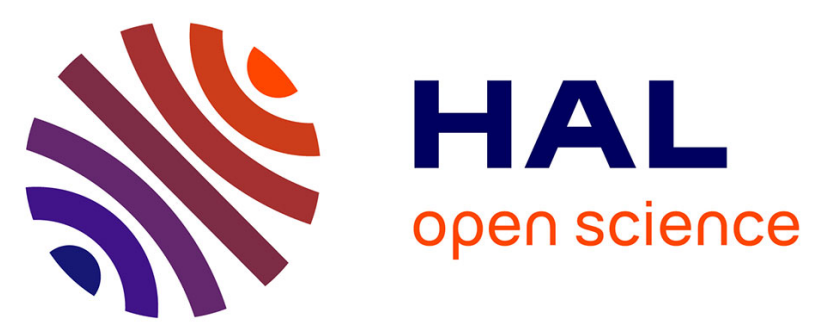

\title{
Le mécanisme de l'extraction dans les sources d'ions positifs à excitation électrique de haute fréquence. Influence d'une induction magnétique longitudinale auxiliaire
}

Serge Vacquie, Jean Bacri, Pierre Benoit-Cattin, Daniel Blanc, René Dagnac, Henri Faye

\section{To cite this version:}

Serge Vacquie, Jean Bacri, Pierre Benoit-Cattin, Daniel Blanc, René Dagnac, et al.. Le mécanisme de l'extraction dans les sources d'ions positifs à excitation électrique de haute fréquence. Influence d'une induction magnétique longitudinale auxiliaire. Revue de Physique Appliquée, 1966, 1 (4), pp.282-286. 10.1051/rphysap:0196600104028200 . jpa-00242734

HAL Id: jpa-00242734

https://hal.science/jpa-00242734

Submitted on 1 Jan 1966

HAL is a multi-disciplinary open access archive for the deposit and dissemination of scientific research documents, whether they are published or not. The documents may come from teaching and research institutions in France or abroad, or from public or private research centers.
L'archive ouverte pluridisciplinaire HAL, est destinée au dépôt et à la diffusion de documents scientifiques de niveau recherche, publiés ou non, émanant des établissements d'enseignement et de recherche français ou étrangers, des laboratoires publics ou privés. 


\title{
LE MÉCANISME DE L'EXTRACTION DANS LES SOURCES D'IONS POSITIFS A EXCITATION ÉLECTRIQUE DE HAUTE FRÉQUENCE. INFLUENCE D'UNE INDUCTION MAGNÉTIQUE LONGITUDINALE AUXILIAIRE.
}

\author{
Par Serge VACQuie, Jean Bacri, Pierre Benoit-CatTin, Daniel Blanc, \\ René DAGNAC et Henri FAYE, \\ Centre de Physique Atomique et Nucléaire, Faculté des Sciences de Toulouse.
}

\begin{abstract}
Résumé. - On étudie des sources du type Thonemann, à couplage inductif. L'emploi d'une sonde mobile a permis de mesurer l'angle de divergence du faisceau extrait, et sa densité ionique, en fonction des divers paramètres caractéristiques de la source. Une induction magnétique longitudinale diminue très considérablement la divergence angulaire du faisceau et augmente la densité du courant extrait, à condition que sa valeur soit convenablement choisie.
\end{abstract}

\begin{abstract}
Thonemann-type sources, with inductive coupling, are studied. With a movable probe, it is possible to make measurements of the divergence angle and of the ionic density of the extracted beam, as a function of the various parameters of the source. A coaxial magnetic induction very much decreases the angular divergence of the beam, and increases the density of the extracted current, if its value is correctly choosen.
\end{abstract}

Plusieurs auteurs [1], [2], [3], [4] ont signalé que, dans des conditions convenables, l'application d'une induction magnétique axiale augmente le courant d'ions positifs produit par les sources à excitation électrique de haute fréquence. L'amélioration est attribuée à une augmentation de la densité du plasma dans la décharge, à l'intérieur de la chambre d'ionisation, mais cette explication est incomplète car on ne saurait négliger l'influence de l'induction magnétique sur le processus d'extraction des ions depuis la source [2]. Cela nous a conduit à étudier l'influence de l'induction magnétique sur l'ouverture angulaire du faisceau et sur sa densité ionique, immédiatement après le canal d'extraction [5], [6].

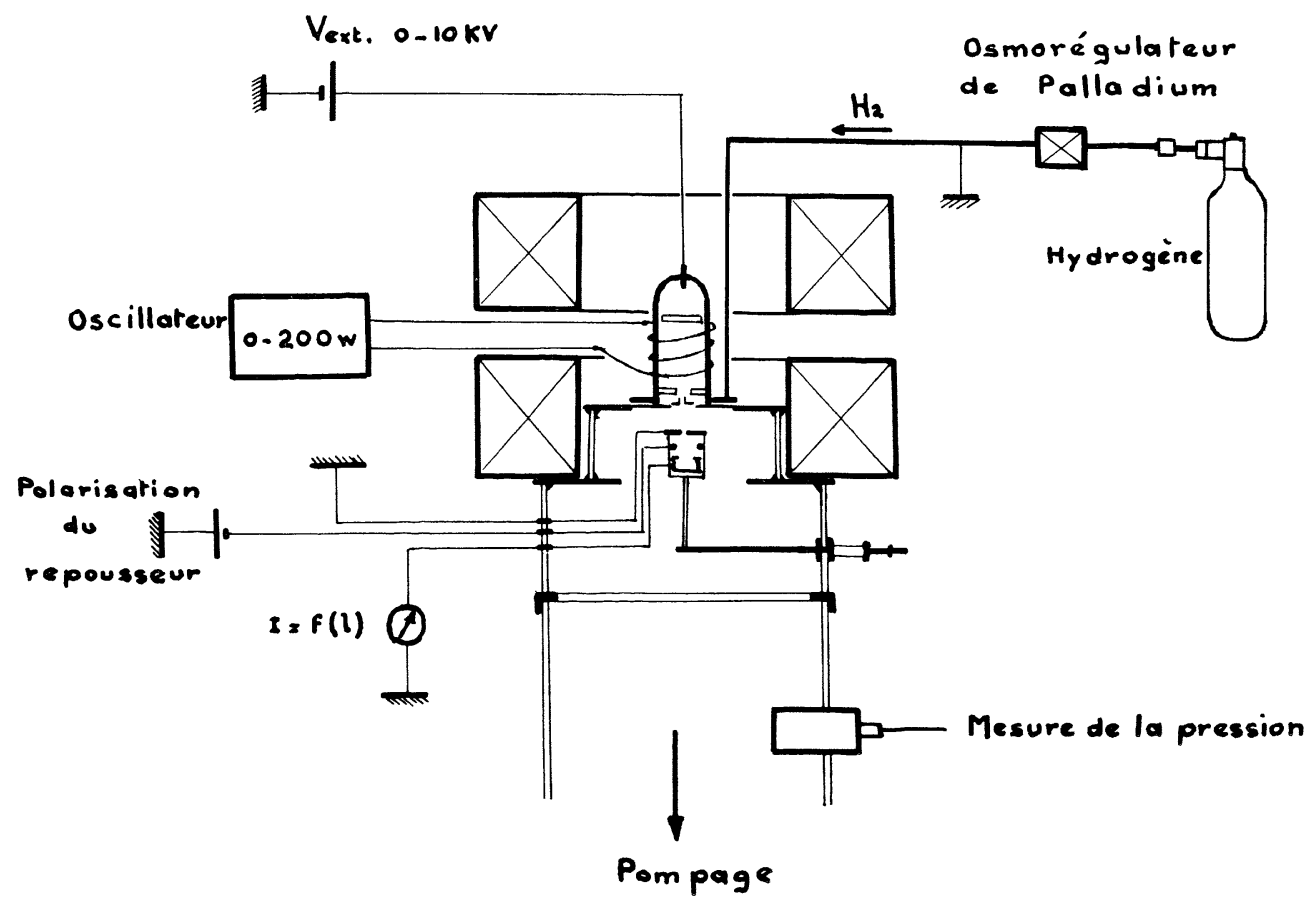

Fig. 1. - Dispositif expérimental. 
Dispositif expérimental. ( $f$ g. 1 et 2). - La source utilisée, du type Thonemann [7], est à couplage inductif. Le système d'extraction est celui mis au point par Blanc et Degeilh [5], [6]. Il est très différent de celui étudié antérieurement par Löb et Peyerimhoff [8]. La source est disposée selon l'axe de deux bobines de Helmholtz, afin que l'induction magnétique soit uniforme dans l'ensemble du volume qu'elle occupe; son intensité peut être réglée de façon continue jusqu'à $8.10^{-2}$ weber $\mathrm{m}^{-2}$.

L'oscillateur à ligne a une fréquence de 195 mégahertz et dissipe une puissance maximale de 200 watts

Le gaz employé est de l'hydrogène ; il est introduit à la base de la source, à travers un osmo-régulateur de palladium. La pression dans la chambre d'ionisation est repérée par une jauge à ionisation, placée au delà, mais préalablement étalonnée par rapport à une jauge de MacLeod mesurant la pression directement dans la chambre.

On explore le faisceau extrait selon un diamètre d'une section droite, grâce à la sonde mobile représentée sur la figure 2: une plaque de dural, percée

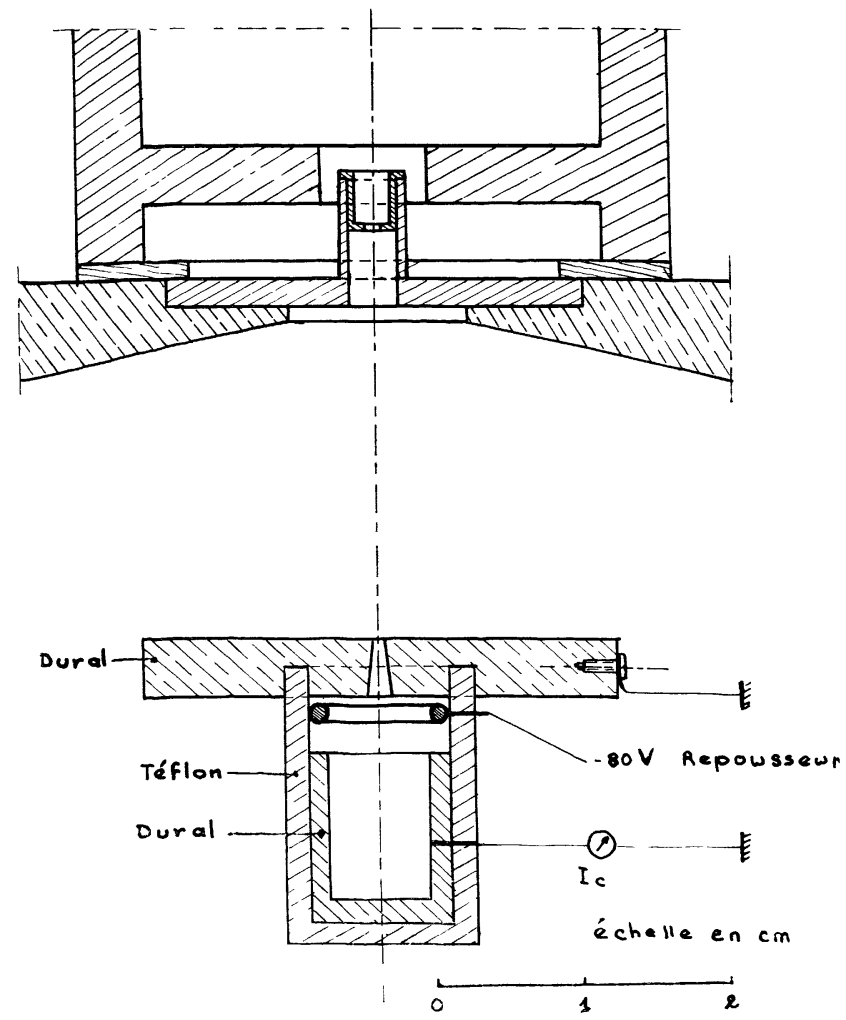

FIG. 2. - Système d'extraction et sonde mobile.

en son centre d'une ouverture circulaire de $1 \mathrm{~mm}$ de diamètre, limite la partie du faisceau recueillie dans le cylindre collecteur. Un anneau polarisé négativement ( -80 volts) élimine la contribution possible des particules secondaires dans le courant recueilli. L'ouverture angulaire $\theta \mathrm{du}$ faisceau se déduit des courbes $I=f(l)$ donnant l'intensité du faisceau en fonction de l'abscisse $l$ de l'axe de la sonde, repérée par rapport à la paroi du système.

Résultats obtenus. - $1^{\circ}$ EN L'ABSENCE D'INDUCtion magnétique. - La figure 3 donne les courbes

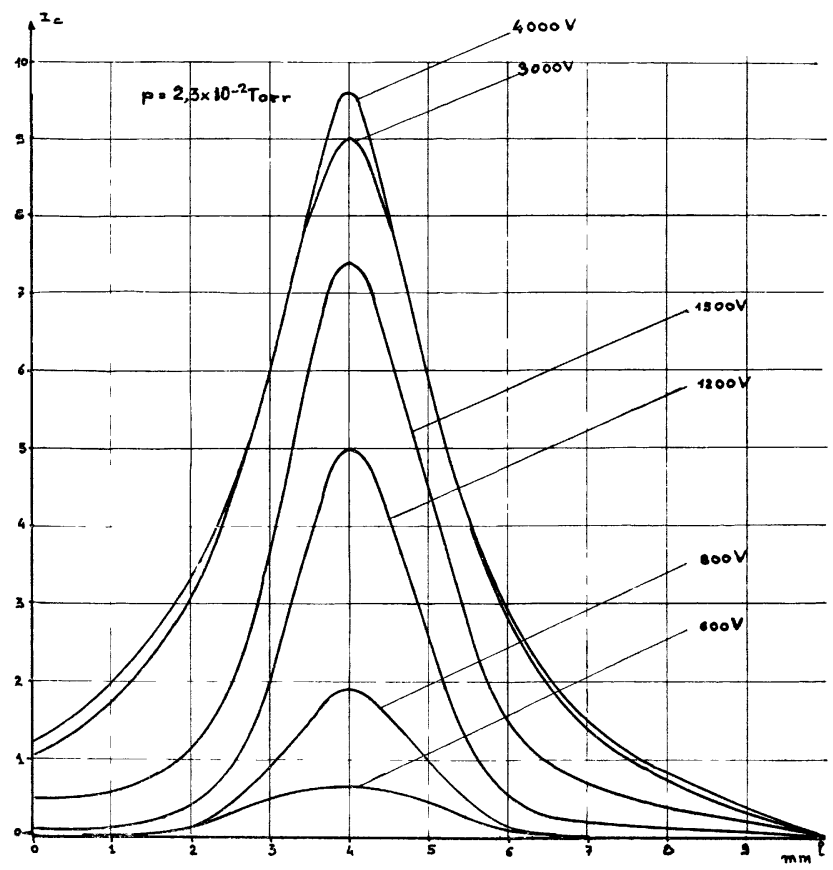

FIG. 3. - Courant recueilli en fonction de la position de la sonde en l'absence de champ magnétique.

$I=f(l)$ pour diverses valeurs de la tension d'extraction, tous les autres paramètres restant constants (pression dans la source : $2,3 \times 10^{-2}$ torr, puissance consommée par l'oscillateur : 150 watts, débit d'hydrogène $10 \mathrm{~cm}^{3} / \mathrm{h}$ ). On en déduit la largeur $L$, au quart de la hauteur de chacune de ces courbes (fig. 4) : elle constitue une assez bonne représentation de la largeur du faisceau. L'allure de la courbe est la même quelle que soit la pression dans la"source; $L$ passe par un minimum ponr une tension de 1000 volts environ, puis par un maximum voisin de 3500 volts.

On peut expliquer ces courbes en considérant le mécanisme de l'extraction ; lorsque la cathode est négative par rapport au plasma, la gaîne ionique qui limite ce dernier prend sensiblement la forme d'une calotte sphérique dont la concavité est dirigée vers le canal d'extraction. La densité $J_{\mathrm{s}} \mathrm{du}$ courant de saturation pouvant atteindre la cathode est donnée par la formule de Child-Langmuir :

$$
J_{\mathrm{s}}=K \cdot \frac{V_{\mathrm{ext}}^{3 / 2}}{x^{2}}
$$

$V_{\text {exl }}$ étant la tension d'extraction, $x$ la distance du 


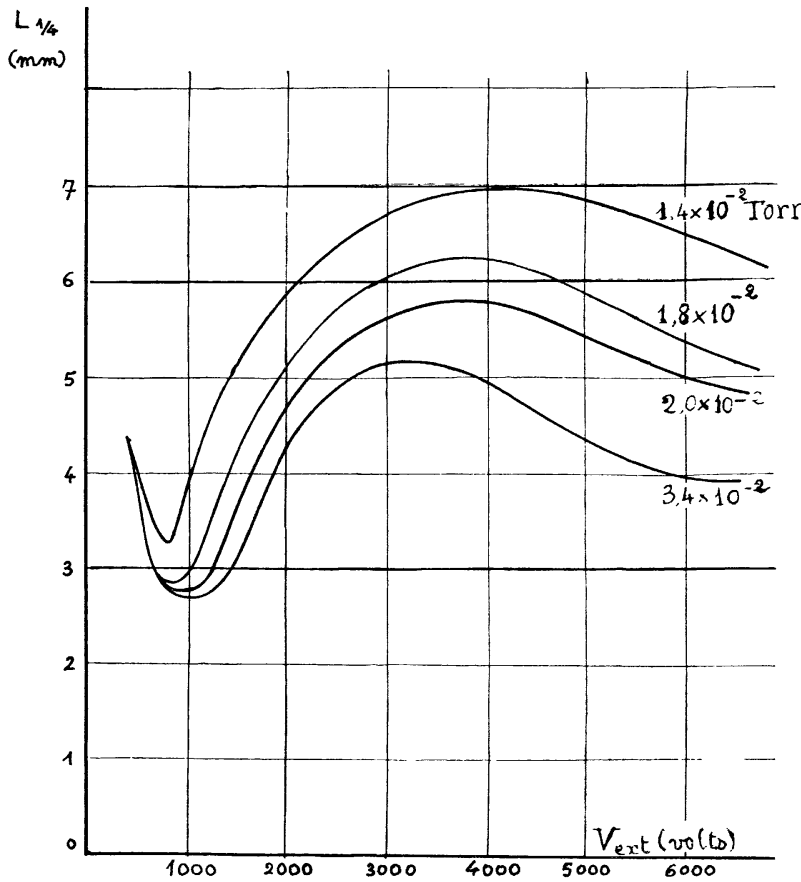

Fig. 4. - Variation de $L$, en fonction de la tension d'extraction, en l'absence de champ magnétique. La pression est prise comme paramètre. point axial de la gaine à la cathode. Cette formule peut être considérée comme une relation entre la tension d'extraction et l'épaisseur de la gaîne, tous les autres paramètres restant constants, ce qui veut dire que, lorsque la tension d'extraction augmente, la limite du plasma s'éloigne de la cathode, ce qui modifie considérablement les propriétés focalisatrices du système d'extraction, comme le montre la figure 5 .

En effet, lorsque $V_{\text {ext }}$ croît de 1000 à 2000 volts, le foyer, qui se plaçait dans la partie inférieure du canal d'extraction, remonte à l'intérieur de celui-ci (fig. 5-1), et l'ouverture angulaire du faisceau augmente, jusqu'à une valeur limite définie par la géométrie du système d'extraction (fig. 5-2).

Pour $V_{\text {ext }}=3000$ volts, le foyer se trouve audessus du diaphragme du canal, et le faisceau se trouve intercepté en partie par ce diaphragme (fig. 5-3) : l'ouverture angulaire diminue.

Au-dessous de 1000 volts, le phénomène ne peut plus être expliqué par des considérations simples d'optique ionique; l'élargissement du faisceau que l'on constate alors est explicable par une diffusion latérale provenant de la faible énergie cinétique des ions.

$2^{\circ}$ EN PRÉSENCE D'UNE INDUCTION MAGNÉTIQUE LONGITUDINALE. - Les trois paramètres dont nous avons étudié l'influence sont l'intensité de l'induc-
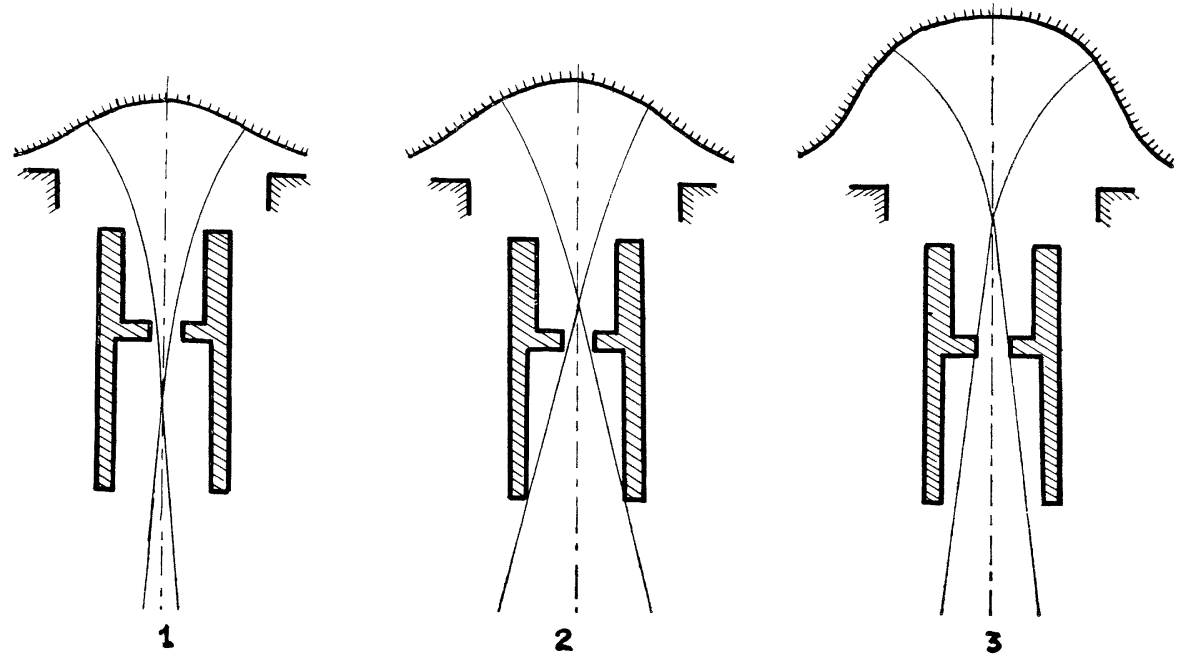

FIG. 5. - Évolution des propriétés focalisatrices du système d'extraction avec la tension d'extraction $\left(V_{1}, V_{2}, V_{3}\right)$.

tion magnétique $B$, la tension d'extraction $V_{\text {ext }}$ et la pression $\mathrm{p}$ à l'intérieur de la source.

La figure 6 présente les courbes $I=f(l)$ pour $V_{\text {ext }}=2000$ volts, $p=2,3 \times 10^{-2}$ torr, et trois valeurs caractéristiques de $B$. L'intensité du faisceau passe par un maximum pour une induction voisine de $1,8 \times 10^{-2}$ weber $\mathrm{m}^{-2}$; la largeur du faisceau est alors minimale ( $\theta$ est voisin de $\left.10^{\circ}\right)$. Pour des valeurs de $B$ plus grandes, l'intensité diminue, mais l'ouverture angulaire du faisceau reste toujours inférieure à sa valeur en l'absence de l'induction magnétique $\left(\theta=20^{\circ}\right)$. La figure 7 montre les courbes $L=f\left(V_{\text {ext }}\right)$ pour trois valeurs caractéristiques de $B$.

On trouvera sur la figure 8 les courbes donnant $L$ en fonction de $B$ pour $p=2,3 \times 10^{-2}$ torr, et trois valeurs de $V_{\text {ext. }}$. On peut distinguer trois régions de fonctionnement distinctes : 


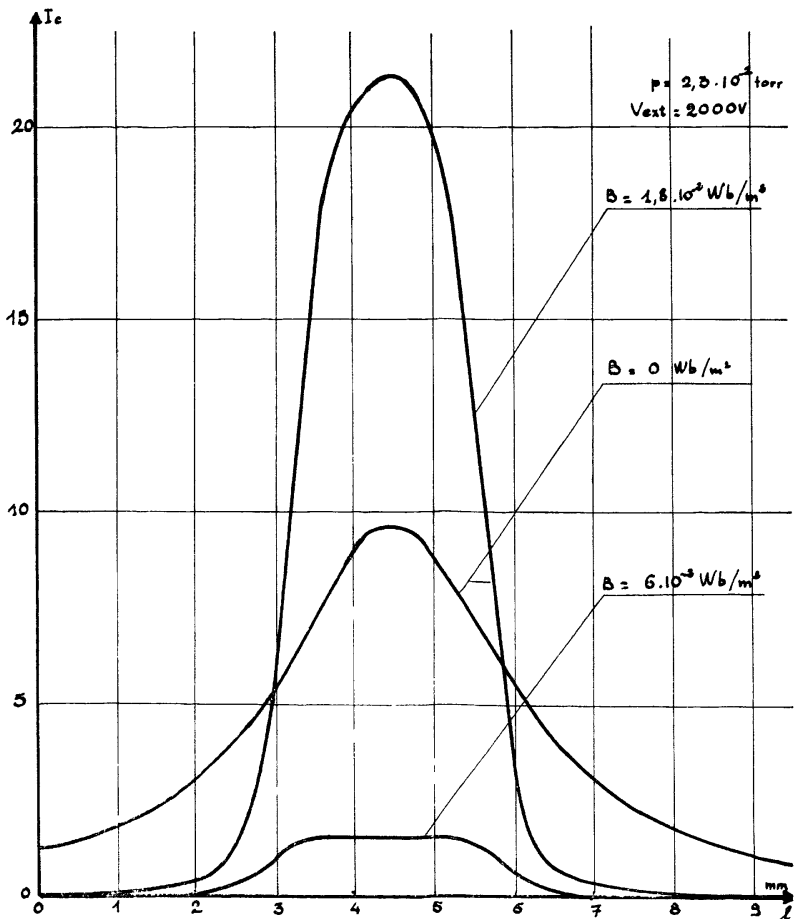

FIG. 6. - Courbes $I=f(l)$ pour trois valeurs du champ magnétique.

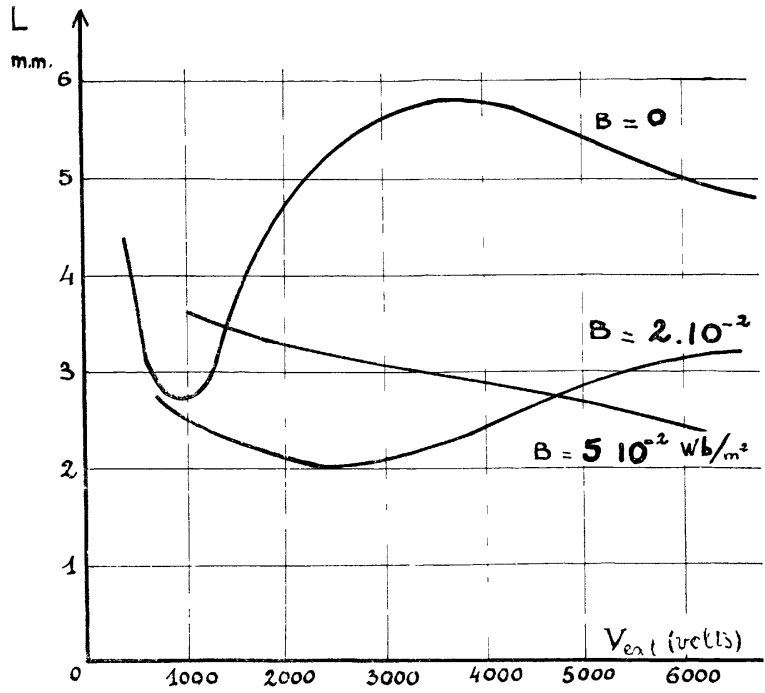

FIg. 7. - Variation de $L$ en fonction de $V_{\text {ext. }}$ Le champ magnétique est en paramètre. $p=2,3 \times 10^{-2}$ torr.

a) Pour $B=10^{-2}$ weber $\mathrm{m}^{-2}$, l'induction a une intensité trop faible pour avoir une influence sensible.

b) Pour $10^{-2} \mathrm{Wbm}^{-2}<B<2 \times 10^{-2}$ weber $\mathrm{m}^{-2}$ l'induction produit une forte concentration ionique selon l'axe du faisceau et, ce resserrement s'accompagne d'une forte diminution de l'ouverture angulaire, qui passe par une valeur minimale.

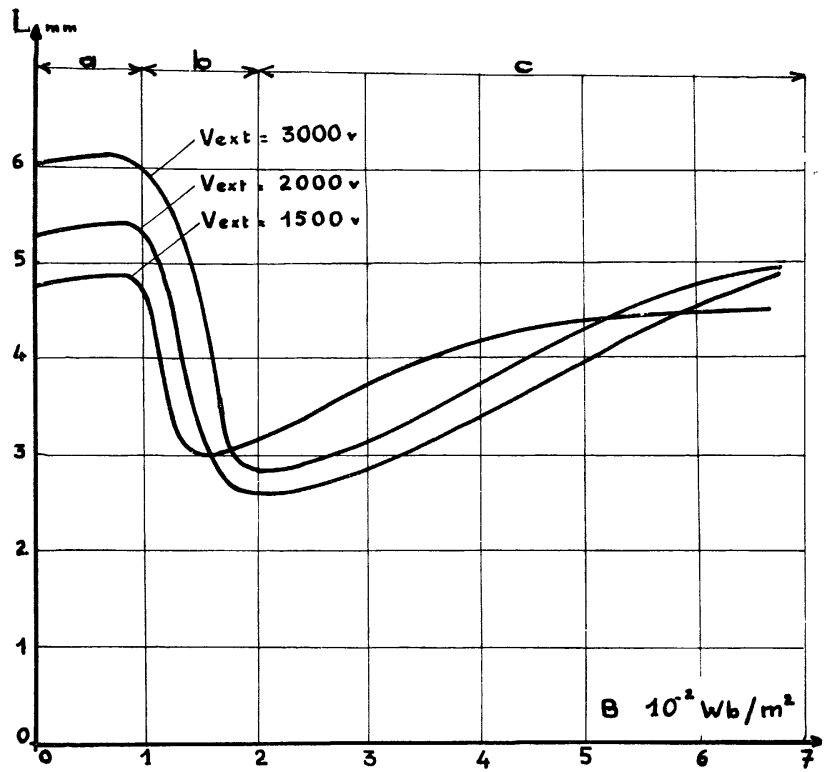

Fig. 8. - Variation de $I$, en fonction du champ magnétique. La tension d'extraction est en paramètre.

$$
p=2,3 \times 10^{-2} \text { torr }
$$

c) Pour $B>2 \times 10^{-2}$ weber $\mathrm{m}^{-2}$, l'intensité du faisceau décroît très rapidement, mais le resserrement selon l'axe demeure et l'ouverture angulaire reste voisine de sa valeur minimale.

Il est impossible de faire une interprétation simple de ces résultats, car l'induction magnétique produit des phénomènes très complexes. Il faut cependant souligner que $B$ agit à deux niveaux :

$A$ l'intérieur du plasma. - Le coefficient de diffusion transversale étant inversement proportionnel à $B^{2}$, les pertes de porteurs de charge sur les parois latérales, par recombinaison, sont plus faibles : la densité du plasma augmente, et l'épaisseur de la gaine ionique le limitant diminue.

Au total, le foyer ( $f$ g. 5) se déplace vers le bas du canal d'extraction, et $\theta$ diminue.

Au niveau de l'extraction. - Lors d'une augmentation continue de $B$, nous avons observé que la gaine qui limite le plasma se rapproche de la cathode. De plus, pour des valeurs de $B$ comprises entre $2,5 \times 10^{-2}$ et $3 \times 10^{-2}$ weber $\mathrm{m}^{-2}$, une émission secondaire très intense d'électrons se produit depuis la cathode ; ces électrons sont accélérés vers l'anode qui peut recevoir un courant assez intense pour provoquer la rupture du bouclier de pyrex qui la protège. Ces électrons modifient d'une façon complexe la distribution du potentiel dans la région comprise entre le plasma et la cathode.

Pour des valeurs plus grandes de $B$, la courbure de la gaine devient très faible : la surface émettrice diminue, ce qui produit une diminution parallèle du courant ionique extrait; le faisceau est alors presque 
cylindrique, ce qui explique la densité presque uniforme mesurée dans toute sa section.

Conclusion. - Nous avons établi quel est le mode global d'action d'une induction magnétique longitudinale sur l'extraction des ions depuis une source à couplage inductif ; il y a diminution de l'ouverture angulaire et augmentation de la densité ionique, pour une valeur convenable de $B$. Il faut noter que ce qui précède n'est valable que si $B$ est effectivement uniforme dans l'ensemble du volume occupé par la source.
L'emploi des sources H. F. était limité par la forte divergence du faisceau extrait, qui rendait difficile l'emploi de lentilles de focalisation électrostatique [9]. L'adjonction d'une induction magnétique élimine totalement cet inconvénient sérieux.

Nous remercions vivement M. André Degeilh, Maître de Conférences à la Faculté des Sciences de Toulouse, avec qui nous avons eu de nombreuses et fructueuses discussions sur ce sujet.

Manuscrit reçu le 11 janvier 1966.

\section{BIBLIOGRAPHIE}

[1] Rutherglen (J. G.) et Cole (J. F. I.), Nature, 1947, $160,545$.

[2] Moak (G. D.), Reese (H.) et Good (W. H.), Nucleonics, 1951, 9, no 3, 18.

[3] Neuert (H.), Z. Naturforsch., 1949, 4a, 449.

[4] Krammer (G.) et alii, Nucl. Instr. and Methods, 1964, 30, 123 .

[5] Degeilh (A.), Thèse Doct. ès-Sciences, Toulouse, 1962, no 191.
[6] Branc (D.) et Degrilli (A.), C. R. Acad. Sc., 1959, 248, 164i.

[7] Thonemann (P. C.), Progr. Nucl. Phys., 1953, 3, 219.

[8] Loв (H.) et Peyerimhof (S.), Z. Naturforsch., 1961, 16-a, 621.

[9] Blanc (D.) et Degeilh (A.), C. R. Acad. Sc., 1961, 253, 1682. 